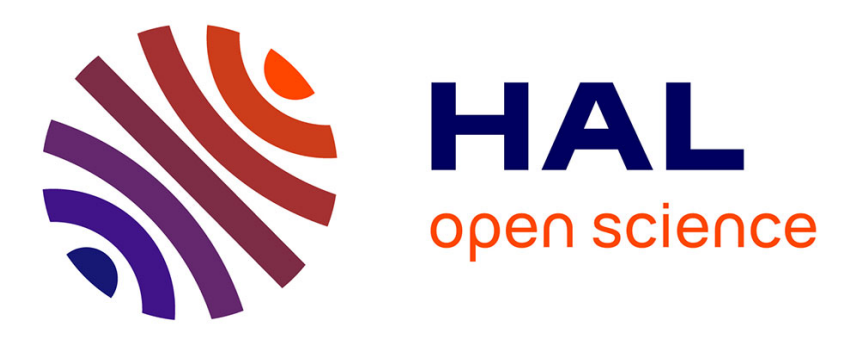

\title{
Capacitive detection of buried interfaces by a dynamic surface force apparatus.
}

\author{
Richard Villey, Agnès Piednoir, Preeti Sharma, Cécile Cottin-Bizonne, \\ Benjamin Cross, Magali Phaner-Goutorbe, Élisabeth Charlaix
}

\section{To cite this version:}

Richard Villey, Agnès Piednoir, Preeti Sharma, Cécile Cottin-Bizonne, Benjamin Cross, et al.. Capacitive detection of buried interfaces by a dynamic surface force apparatus.. Review of Scientific Instruments, 2013, 84 (8), pp.085113. 10.1063/1.4817639 . hal-01088110

\section{HAL Id: hal-01088110 https://hal.science/hal-01088110}

Submitted on 5 Apr 2019

HAL is a multi-disciplinary open access archive for the deposit and dissemination of scientific research documents, whether they are published or not. The documents may come from teaching and research institutions in France or abroad, or from public or private research centers.
L'archive ouverte pluridisciplinaire HAL, est destinée au dépôt et à la diffusion de documents scientifiques de niveau recherche, publiés ou non, émanant des établissements d'enseignement et de recherche français ou étrangers, des laboratoires publics ou privés. 


\section{AIP scientifiti instruments}

Capacitive detection of buried interfaces by a dynamic surface force apparatus

Richard Villey, Agnès Piednoir, Preeti Sharma, Cécile Cottin-Bizonne, Benjamin Cross et al.

Citation: Rev. Sci. Instrum. 84, 085113 (2013); doi: 10.1063/1.4817639

View online: http://dx.doi.org/10.1063/1.4817639

View Table of Contents: http://rsi.aip.org/resource/1/RSINAK/v84/i8

Published by the AIP Publishing LLC.

Additional information on Rev. Sci. Instrum.

Journal Homepage: http://rsi.aip.org

Journal Information: http://rsi.aip.org/about/about_the_journal

Top downloads: http://rsi.aip.org/features/most_downloaded

Information for Authors: http://rsi.aip.org/authors 


\title{
Capacitive detection of buried interfaces by a dynamic surface force apparatus
}

\author{
Richard Villey, ${ }^{1}$ Agnès Piednoir, ${ }^{1}$ Preeti Sharma, ${ }^{2}$ Cécile Cottin-Bizonne, ${ }^{1}$ \\ Benjamin Cross, ${ }^{2}$ Magali Phaner-Goutorbe ${ }^{3}$ and Élisabeth Charlaix ${ }^{2}$ \\ ${ }^{1}$ Institut Lumière Matière, Université Lyon 1, CNRS, F-69622 Villeurbanne, France \\ ${ }^{2}$ Laboratoire Interdisciplinaire de Physique, Université Joseph Fourier, F-38402 Saint Martin d'Hères, France \\ ${ }^{3}$ Institut des Nanotechnologies de Lyon, École Centrale de Lyon, F-69130 Écully, France
}

(Received 18 June 2013; accepted 22 July 2013; published online 13 August 2013)

\begin{abstract}
We present here a new type of distance sensor mounted on a Surface Force Apparatus (SFA), able to detect the position of a buried interface and therefore the thickness of a thin solid or soft matter film coating the SFA surface(s). This sensor relies on the capacitance created by the two metallized surfaces of the SFA. An harmonic oscillation of these polarized surfaces creates a pico- to femto-amps current indicating their relative position. One of the specificities of this sensor is the relatively weak polarization used for the measurements, minimizing the electrical forces and their impact on other interactions, hydrodynamical and mechanical forces measured by the SFA. This original and simple design is of high interest for studying the viscoelastic properties of thin films, to detect adsorbed liquid layers or slippage at liquid-solid interfaces, or even to study complex fluids such as ionic liquids under polarization. We demonstrate the use of this sensor to study the flow boundary condition of silicon oil on a metal surface, and the elastic modulus of a thin elastomer layer. @ 2013 AIP Publishing LLC. [http://dx.doi.org/10.1063/1.4817639]
\end{abstract}

\section{INTRODUCTION}

Detecting interfaces is always a critical part of the study of confined systems. This is especially important for nanoscale rheological and mechanical measurements, which are essential for tribological applications as well as in microand nanofluidic systems. ${ }^{1}$ Most of the mechanical calculations which relate forces and displacements to mechanical properties, assume that the position of boundaries are perfectly known. However, if local force measurement devices such as the surface force balance or the Atomic Force Microscope (AFM) may be able to detect the outer surface of a sample from the strong repulsive force arising at contact, inner interfaces, such as the buried interface between a supporting substrate and a thin coating layer, are more difficult to access. For example, when studying hydrodynamic forces in a thin liquid film, it is of crucial importance to locate the solidliquid interface in order to determine the actual flow boundary condition. ${ }^{2-4}$ However this is not always possible if liquid layers are strongly adsorbed on the solid surface and are not removed by the contact force. ${ }^{5,6}$ The characterization of such immobilized layers, and more generally the details of flow properties at a solid interface, is of high importance for lubrication issues, or for the electrokinetic transport properties arising in nanofluidic systems. ${ }^{7,8}$ Another example is the mechanical properties of thin organic or biological films supported by a more rigid substrate, such as polymer brushes on silicon. Here it is well known that the effective stiffness of the film depends utterly on its thickness. ${ }^{9,10}$ Therefore, the detection of the interface between the supporting substrate and the coating layer is often the limiting parameter for the determination of the layer mechanical properties.

The surface force apparatus (SFA) is a well-suited device to study complex rheological properties of interfaces and/or thin film mechanical properties. ${ }^{1}$ We have developed over the years an original design for a dynamic-mode SFA where static and oscillating forces between a sphere and a plane are measured together with the relative sphere-plane displacement. ${ }^{11,12}$ The oscillating forces come from a nanometric oscillation of the sphere, which creates a drainage flow at the same frequency within a thin liquid film of tunable thickness inserted between the sphere and the target surface. Together with the equilibrium interaction force, the hydrodynamic impedance (defined by the real and imaginary part of the dynamic force divided by the sphere oscillation amplitude) provides a detailed characterization of the interfacial mechanics. ${ }^{13,14}$

Here, we describe an in situ absolute distance sensor based on capacitance measurements, able to measure the distance between the metallic surfaces of the SFA without the need of putting them into contact. This sensor can be used to detect the buried interface between the metal surface and a thin film coating it. Capacitance measurements have been used in weak force measurements such as the Casimir-Van der Waals forces in order to detect long-range distances between metallic surfaces. ${ }^{15,16}$ However, the disadvantage of this kind of measurement is the generation of an electrical force which can interfere with other interaction forces. Here, we use a simple method allowing a relatively low polarization, with a minimal impact on both the static and the dynamic forces. The principle of the sensor is presented in Sec. II and its implementation on the SFA in Sec. III. Section IV discusses the resolution of the sensor and its impact on the dynamic forces measurements in two cases of interest: the hydrodynamic boundary condition of silicon oil on a metal surface, and the non-contact determination of the Young's modulus of a soft elastomer film using hydrodynamic impedance measurements. 


\section{PRINCIPLE OF THE SPHERE-PLANE ABSOLUTE DISPLACEMENT SENSOR}

The absolute distance sensor described here is based on the measurement of the derivative of the capacitance between a metallic sphere and a metallic plane. The capacitance $C_{\mathrm{SP}}$ between a plane and a sphere of radius $R$ made of a perfect conductor and separated by a distance $D_{\mathrm{EL}}$ (at the apex of the sphere) is given by ${ }^{17}$

$$
C_{\mathrm{SP}}\left(D_{\mathrm{EL}}\right)=2 \pi \epsilon R\left[\ln \left(\frac{R}{D_{\mathrm{EL}}}\right)+\ln (2)+\frac{23}{20}+\kappa\right],
$$

where $\kappa$ is a constant between 0 and 0.02 . This formula holds if $R \gg D_{\mathrm{EL}}$ and if the gap has a uniform dielectric constant value $\epsilon$. In the configuration studied below, the fluid phase between the sphere and the plane has a uniform dielectric constant, but one or eventually both surfaces may be coated with a layer of thickness $e_{i}\left(i=1\right.$ or 2) and dielectric constants $\epsilon_{i}$. In this case, $D_{\mathrm{EL}}$ in Eq. (1) and all equations hereafter should be replaced by $D_{\mathrm{EL}}+\sum_{i} e_{i}\left(\epsilon-\epsilon_{i}\right) / \epsilon_{i}$. In the following we keep the notation $D_{\mathrm{EL}}$ for the sake of simplicity.

In the case of a typical SFA experiment, with a millimetric sphere and a micro- to nano-metric gap filled with an organic liquid $\left(\epsilon \sim 2 \epsilon_{0}\right)$, the capacitance value is in the $1-10 \mathrm{pF}$ range. With such a low value, parasitic capacitances become easily predominant; moreover, $C_{\mathrm{SP}}$ is a slowly varying function of $D_{\mathrm{EL}}$. Thus, it is of interest to focus on the inverse of the derivative of the sphere-plane capacitance with respect to the distance:

$$
\frac{d D_{\mathrm{EL}}}{d C_{\mathrm{SP}}}=\frac{D_{\mathrm{EL}}}{2 \pi \epsilon R} .
$$

This relation gives a direct access to the distance $D_{\mathrm{EL}}$ (eventually augmented by the offset due to the presence of dielectric coatings) between the conductive surfaces.

In a dynamic SFA, the dynamics of interfacial properties are studied by oscillating the liquid gap width at a low amplitude $D(t)=D+h_{0} \cos (\omega t)$. Here, we take advantage of this oscillation to measure the derivative of the sphereplane capacitance (Eq. (2)). For this purpose, we apply a constant polarization V as sketched in Fig. 1 and use a lockin amplifier to measure the intensity and phase of the current $I(t)=V d C_{\mathrm{SP}} / d t$ feeding the capacity change. For an ideal circuit (negligible resistance and inductance) we expect $I(t)=\operatorname{Re}[\tilde{I} \exp (j \omega t)]$ with a complex amplitude:

$$
\tilde{I}=-j h_{0} \omega V \frac{d C_{\mathrm{SP}}}{d D_{\mathrm{EL}}}=-j h_{0} \omega V \frac{2 \pi \epsilon R}{D_{\mathrm{EL}}} .
$$

Thus, the plot of $h_{0} / \operatorname{Im}(\tilde{I})$ as a function of $D_{\mathrm{EL}}$ should be a straight line whose origin gives the position of the electric contact.

However, the applied polarization has an incidence on the balance of interfacial forces and on mechanical impedance measurements. The polarization $V$ creates a long-range electrostatic force

$$
F_{\mathrm{EL}}=-\frac{\pi \epsilon R V^{2}}{D_{\mathrm{EL}}}
$$

As an example, for a $1 \mathrm{~V}$ polarization this force at $D_{\mathrm{EL}}=10$ $\mathrm{nm}$ is in the tenth of $\mu \mathrm{N}$ (with a sphere radius of $3 \mathrm{~mm}$ ).

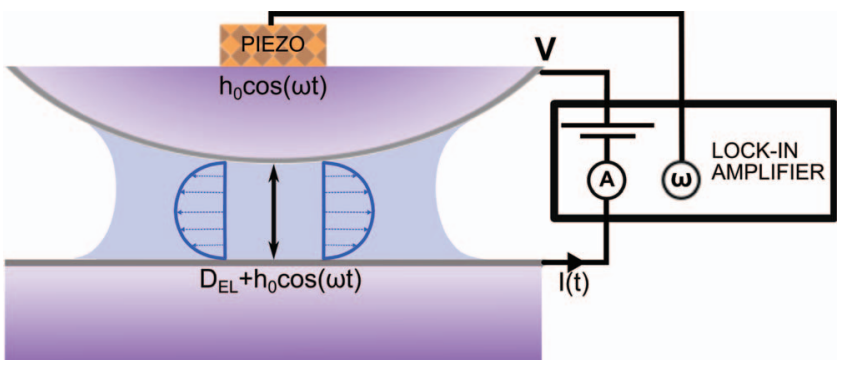

FIG. 1. Principle of the absolute displacement sensor. A metal-coated sphere is placed in front of a metal coated plane, forming a capacitor of capacitance $C_{\mathrm{SP}}$. The gap of minimum value $D_{\mathrm{EL}}$ is filled by a dielectric liquid. The mechanical oscillation of the plane at frequency $f=\omega / 2 \pi$ creates an oscillation of the gap width. The capacitance is thus oscillating, creating a current $\tilde{I}$, which is measured using a lock-in amplifier.

For comparison, the very weak Van der Waals interaction in liquids, $F_{V d W}=-A R / 6 D^{2}$ (with $A$ being the Hamaker constant) is in the $100 \mathrm{nN}$ at the same distance. It is thus of interest to keep the polarization as low as possible.

The polarization also impacts the mechanical impedance

$$
Z(\omega, D)=\tilde{F}_{\mathrm{ac}} / h_{0}=Z^{\prime}+j Z^{\prime \prime},
$$

measured from the complex amplitude $\tilde{F}_{\text {ac }}$ of the force at the frequency of the harmonic oscillation, by creating an unwanted component on the real part of $\mathrm{Z}$ :

$$
Z_{\mathrm{EL}}=-\frac{\pi \epsilon R V^{2}}{D_{\mathrm{EL}}^{2}} .
$$

This parasitic mechanical impedance is directly proportional to the square of the electric field. With the above values, it is about $1 \mu \mathrm{N} / \mathrm{nm}$ at $D_{\mathrm{EL}}=10 \mathrm{~nm}$, to be compared to the hydrodynamic impedance of a liquid of low viscosity, of about $40 \mu \mathrm{N} / \mathrm{nm}$ at $20 \mathrm{~Hz}$. It is thus important to keep the electric field lower than $10^{8} \mathrm{~V} / \mathrm{m}$. A usually more restrictive condition is that the field should not exceed the dielectric strength of the materials, of order of $10^{7} \mathrm{~V} / \mathrm{m}$ for many organic materials.

For a polarization of $1 \mathrm{~V}$, a ratio $h_{0} / D_{\mathrm{EL}}=3 \%$, and a frequency $\omega / 2 \pi=20 \mathrm{~Hz}$, the current amplitude is around 1.2 $\mathrm{pA}$. This requires a current measurement with a noise at most of some tens of fA. We have chosen here to use the input for current measurement of a lock-in amplifier (SR830, Stanford Research System) without signal pre-amplification to directly realize the very simple electric wiring of Fig. 1.

\section{IMPLEMENTATION ON A DYNAMIC SFA}

The dynamic SFA used is described in Ref. 11. A sphere is moved in a direction normal to a plane (the target surface) using two piezoelectric elements. One element changes very slowly the mean sphere-plane distance, while the other one is excited harmonically with a low amplitude at frequency $\omega / 2 \pi$, by a lock-in amplifier SR830. The relative displacement between the sphere and plane $D+h_{0} \cos (\omega t)$ is measured through an external sensor. The plane is mounted on a double-beam cantilever whose deflection is measured through an interferometric sensor and gives access to the interaction force $F_{\mathrm{dc}}+\operatorname{Re}\left[\tilde{F}_{\mathrm{ac}} \exp (j \omega t)\right]$. The sensors have respectively a displacement resolution of $0.1 \mathrm{~nm}$ (quasistatic) and $5 \mathrm{pm}$ 
(dynamic at $19 \mathrm{~Hz}$ ), and a force resolution of $700 \mathrm{nN}$ (quasistatic) and $10 \mathrm{nN}$ (dynamic at $19 \mathrm{~Hz}$ ).

The quasistatic components $D$ and $F_{\mathrm{dc}}$ are obtained by low-pass filtering the corresponding signals, while the harmonic components $h_{0}$ and $\widetilde{F}_{\text {ac }}$ are analyzed by two double phase lock-in amplifiers SR830 (the harmonic displacement providing the phase reference). We use here a third SR830 to polarize the surfaces (using a dc output) and to measure the amplitude and phase of the oscillating current at the frequency $\omega / 2 \pi$.

In the following experiments, the conductive sphere and plane are made of pyrex coated with a metal layer. The plane is a float borosilicate (Pignat), and the sphere of radius $R \approx 3$ $\mathrm{mm}$ is obtained by melting a borosilicate bar as described in Ref. 11. The metal coating consists of a $5 \mathrm{~nm}$ Chromium layer covered by a $30 \mathrm{~nm}$ Platinum layer, both of them deposited using magnetron sputtering. Platinum is chosen to prevent the formation of an oxide layer. The final roughness is less than $0.5 \mathrm{~nm}$ on a $65 \mu \mathrm{m} \times 65 \mu \mathrm{m}$ area. Many precautions (preparation under laminar flow cabinet and storage in a vacuum chamber) are taken to avoid any contamination by small dusts which could prevent access to small values of sphere-plane separation $D$. We use the following heuristic criterion: there should be at maximum 1 particle of height equal or larger than $10 \mathrm{~nm}$ in a $20 \mu \mathrm{m} \times 20 \mu \mathrm{m}$ area on both the sphere and plane. This criterion comes from the fact that if there is 1 particle of $10 \mathrm{~nm}$ or more at a distance of $10 \mu \mathrm{m}$ or less from the apex of a $3 \mathrm{~mm}$ radius sphere, then the value $D=0 \mathrm{~nm}$ cannot be reached without squeezing this particle.

The electric contact is made after mounting the surfaces on a part of the SFA isolated from the electric ground. Electric isolation is assured by mounting the plane on a metallic plane holder which is itself mounted on a ceramic bar. The metallized sphere is connected to the circuit using silver painting (LeitSilber, Ted Pella) and two very flexible wires to prevent unwanted mechanical forces transmission. The $5 \mathrm{~cm}$ long flexible wires are connected to the cores of two coaxial cables whose shields are connected to the mass of the SR830. One core is connected to the current input of the lock-in, the other one to the dc output of the same lock-in. All electric contacts are to be thoroughly taken care of to avoid electromagnetic interferences.

The confined liquid used is a silicon oil (Rhodorsil Oil 47 V20, Bluestar Silicones) with a relative dielectric constant of 2.68 and a dielectric strength of $1.4 \times 10^{7} \mathrm{~V} / \mathrm{m}$. The oil is degassed and filtered with a $0.2 \mu \mathrm{m}$ porous membrane before use to ensure absence of bubbles and small particles contamination.

\section{RESULTS AND DISCUSSION}

\section{A. Distance measurement}

Figure 2 shows the real and imaginary parts of the current measured as a function of the relative displacement, for a polarization of $2 \mathrm{~V}$ applied to the raw conductive surfaces (no dielectric coating). In this experiment, the level of mechanical oscillation $h_{0}$ is changed between 0.3 and $28 \mathrm{~nm}$ to keep a ratio $h_{0} / D$ less than $3 \%$. The frequency is $\omega / 2 \pi=19 \mathrm{~Hz}$. In



FIG. 2. In phase (blue) and out of phase (red) components of the current measured during a SFA experiment using a $2 \mathrm{~V}$ potential difference between the sphere and the plane. The jumps visible in the out-of-phase component come from the change in the amplitude $h_{o}$ of the mechanical oscillation. The inset shows the noise in $\operatorname{Im}(\tilde{\mathrm{I}})$, which is $11 \mathrm{fA} \mathrm{rms}$.

addition to this harmonic oscillation the sphere is moved very slowly at a velocity between $0.2 \mathrm{~nm} / \mathrm{s}$ (close to contact) and $5 \mathrm{~nm} / \mathrm{s}$ (far from contact) to change the nominal distance. We can see in Fig. 2 that the current response is essentially outof-phase with the mechanical excitation, which shows that the electric circuit is close to the expected one. The inset shows that the noise level on the current is around $11 \mathrm{fA} \mathrm{rms,} \mathrm{low}$ enough to ensure a good distance measurement as calculated in Sec. II. This noise is close to the intrinsic noise of the lockin amplifier SR830. The latter is of $13 \mathrm{fA} / \sqrt{\mathrm{Hz}}$ for the current amplification of $10^{8} \mathrm{~V} / \mathrm{A}$ used in this experiment, giving a noise level of $6.6 \mathrm{fA}$ with a bandwidth of $0.26 \mathrm{~Hz}$. This indicates that the electric contacts are of sufficient quality and that electromagnetic interferences are negligible in this circuit.

Then, we can plot $h_{0} / \operatorname{Im}(\tilde{\mathrm{I}})$ which is expected to be proportional to the distance $D_{\text {EL }}$ between the conductive surfaces, according to Eq. (3). Figure 3(a) plots this quantity as a function of the surface displacement, for 5 different applied polarizations ranging from 0 to $5 \mathrm{~V}$. The plots are straight lines pointing towards zero values of $D_{\mathrm{EL}}$ quite close from each other. We can notice that even at a $0 \mathrm{~V}$ applied polarization there is still a measurable current, which indicates the presence of a residual polarization around $40 \mathrm{mV}$. Therefore, the plots of $h_{0} / \operatorname{Im}(\tilde{I})$ are fitted to Eq. (3) using the polarization $V$ and the zero value or $D_{\mathrm{EL}}$ as adjustable parameters (Table I). The absolute origin for the sphere-plane distance is arbitrarily chosen to be the electrical zero of the $0.5 \mathrm{~V}$ polarization. We can see in Table I that an offset of $20-40 \mathrm{mV}$ is observed for every applied polarization. This reproducible offset is attributed to the presence of a contact polarization of the surfaces. ${ }^{16}$

Apart from this polarization offset, the experimental data of $h_{0} / \operatorname{Im}$ (I) fit perfectly with Eq. (3), and determine electrical origins within $\pm 2 \mathrm{~nm}$ (discarding the polarization of $40 \mathrm{mV}$ ). Since the fittings of the current measurements are appropriate up to more than $4 \mu \mathrm{m}$ we can conclude that the resolution of this distance measurement is better than $\pm 2 \mathrm{~nm}$ at a distance of $4 \mu \mathrm{m}$, even with a polarization as low as $0.5 \mathrm{~V}$. Since the 


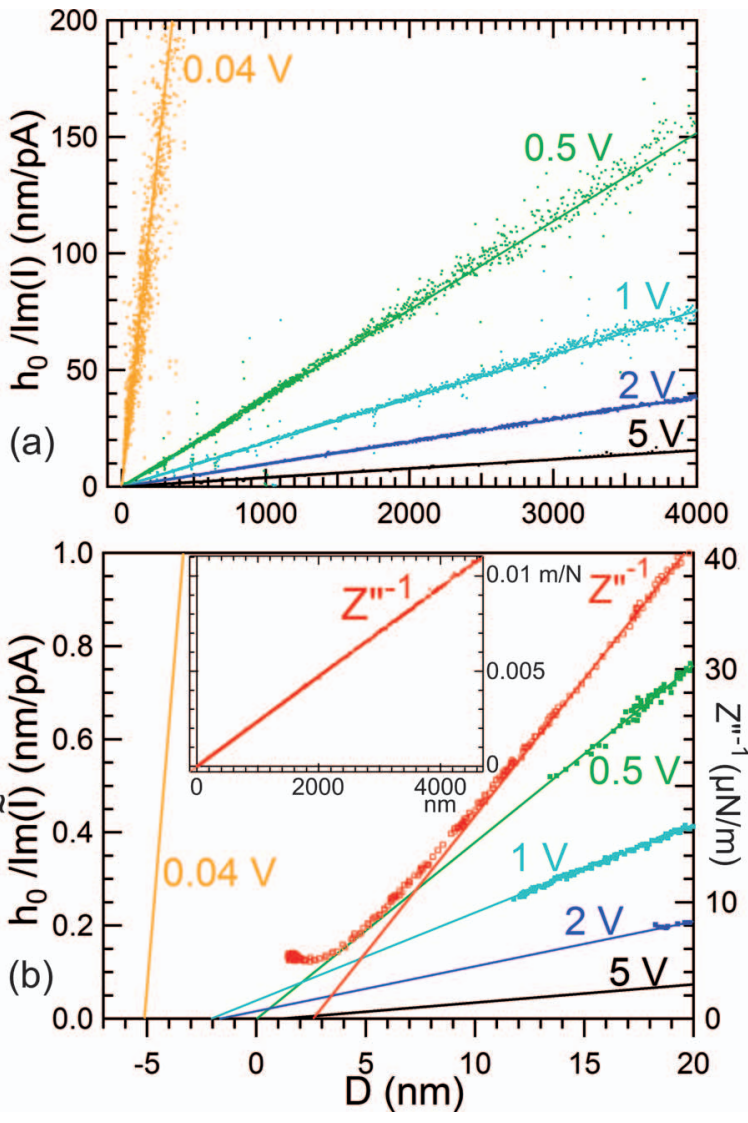

FIG. 3. (a) Plot of $h_{0} / \operatorname{Im}(\tilde{I})$ as a function of the sphere-plane distance, for the $0,0.5,1,2$, and $5 \mathrm{~V}$ polarizations (see Eq. (3)). The distance is measured by the external displacement sensor, and the origin is chosen arbitrarily as the electrical origin given by the $0.5 \mathrm{~V}$ polarization. The continuous straight lines are the best fits of the data with Eq. (3). The fitted polarizations and origin offsets are reported in Table I. (b) Comparison of electric and hydrodynamic origins of distances. The left-axis is an enlargement of (a) around the origin. On the right-axis, data points are the inverse of the hydrodynamic impedance $Z^{\prime \prime-1}=h_{0} / \operatorname{Im}\left(\tilde{\mathrm{F}}_{\mathrm{ac}}\right)$ (Eq. (5)), and the continuous red line is the linear extrapolation of its large distance behavior plotted in inset. The deviation of $Z^{\prime \prime}-1$ from a straight line close to the contact does not depend on the polarization.

current measured is proportional to the electric field $V / D_{\mathrm{EL}}$, this nanometric precision could be achieved with the sphere and plane separated by several tens of micrometers, provided that the applied polarization is high enough. The electric field obtained for the $0.5 \mathrm{~V}$ polarization at a distance of $4 \mu \mathrm{m}$, of some $10^{5} \mathrm{~V} / \mathrm{m}$, gives indeed the lowest field value needed for this electrical distance measurement.

TABLE I. Fitted values for the effective polarization and the position of electric zeros. We have arbitrarily chosen the polarization equal to $0.5 \mathrm{~V}$ as the reference for distance origin. Error bars come from the fits themselves and from the uncertainty on the sphere radius $R=2.86 \pm 0.02 \mathrm{~mm}$.

\begin{tabular}{lcc}
\hline \hline $\begin{array}{l}\text { Applied } \\
\text { polarization (V) }\end{array}$ & $\begin{array}{c}\text { Measured } \\
\text { polarization (V) }\end{array}$ & $\begin{array}{c}\text { Electrical } \\
\text { origin (nm) }\end{array}$ \\
\hline 0 & $0.035 \pm 0.007$ & $-5.2 \pm 6$ \\
0.5 & $0.52 \pm 0.02$ & $\ldots$ \\
1 & $1.04 \pm 0.03$ & $-2.1 \pm 0.2$ \\
2 & $2.04 \pm 0.04$ & $-1.7 \pm 0.1$ \\
5 & $5.03 \pm 0.06$ & $1.2 \pm 0.1$ \\
\hline \hline
\end{tabular}

Conversely, one can notice in Fig. 3(b) the very high electric fields that have been reached without breakdown, up to ten times larger than the breakdown field of $14 \mathrm{mV} / \mathrm{nm}$ indicated by the manufacturer of the silicon oil used. For example, using the $5 \mathrm{~V}$ polarization, a current in accordance with Eq. (3) is still measured at $D=35 \mathrm{~nm}$, corresponding to an electric field larger than $140 \mathrm{mV} / \mathrm{nm}$. Eventually the breakdown should occur, which we prevented by shutting down the sphere-plane polarization at distances below 10-30 nm. Since contamination by dusts can cause premature breakdown, we explain the existence of such high fields without breakdown by the high cleanliness of the surfaces and of the oil.

Figure 3(b) illustrates the use of this capacitance distance measurement to characterize the flow boundary condition of silicon oil on the metal surfaces. For this purpose, we use the imaginary part of the mechanical impedance $Z^{\prime \prime}$. The expression of $Z^{\prime \prime}$ in the case of a no-slip boundary condition is derived from the so-called Reynolds force for the drainage flow between a sphere and a plane: ${ }^{6}$

$$
\frac{1}{\operatorname{Im}(\mathrm{Z}(\omega, \mathrm{D}))}=Z^{\prime \prime-1}=\frac{D_{\mathrm{H}}}{6 \pi \eta R^{2} \omega},
$$

with $\eta$ as the liquid viscosity and $D_{\mathrm{H}}$ as the thickness of the liquid film separating the no-slip boundaries. Figure 3(b) shows that the hydrodynamic origin $\left(D_{\mathrm{H}}=0\right)$ is located at a distance equal to $2.8 \mathrm{~nm}$ from the electrical origin for the polarization $0.5 \mathrm{~V}$, in the liquid phase. This offset is larger than the uncertainty on the electrical origin measurement. According to the results of Georges et al. ${ }^{6}$ we interpret this offset as the presence of "immobile" liquid layers on the solid surfaces, due to chains adsorption on the platinum. The thickness of the "immobile" layer on each surface is thus $1.4 \pm 1 \mathrm{~nm}$, which is somewhat smaller than the radius of gyration of the chains, estimated to $3 \mathrm{~nm}$ (assuming a Gaussian polydimethylsiloxane (PDMS) chain of about 40 monomers of size $0.5 \mathrm{~nm}$ ). This result is in good qualitative agreement with the one of Georges et al. for the boundary flow of polymer melts (poly-isoprene) on cobalt surfaces. ${ }^{6}$

Finally, we present in Fig. 4 the comparison between hydrodynamic and electric origin of distances in the case of a plane coated with an elastomer film made of reticulated PDMS (PDMS SYLGARD 184, Dow Corning). In this case, the conductive plane was created using a conductive silicon wafer (p-doped (111) wafers, 0.1-0.01 $\Omega \mathrm{cm}$, Siltronix) to ensure a good polymer adsorption. The PDMS was diluted in heptane (93\% heptane, $7 \%$ PDMS), deposited on the conductive surface using spin coating at $1000 \mathrm{rpm}$, and then cured at $50{ }^{\circ} \mathrm{C}$ for $24 \mathrm{~h}$. Since no slippage is expected for the PDMS oil on the reticulated PDMS, the hydrodynamic zero should give the top of the elastomer layer and the electric zero its bottom. Thus the apparent thickness of the layer, $\tau=1324 \pm 2 \mathrm{~nm}$, can directly be read in Fig. 4. If oil adsorption exists on the top of the elastomer it should stay at a molecular thickness and not change this value noticeably. The real thickness $e$ of the layer is obtained from its apparent thickness by $e=\tau \epsilon_{1} / \epsilon$. Note that here, the dielectric constant of the reticulated PDMS is 2.65 , so the real thickness of the layer is $e=1309 \pm 7 \mathrm{~nm}$. We have added in the real thickness uncertainty the precision 


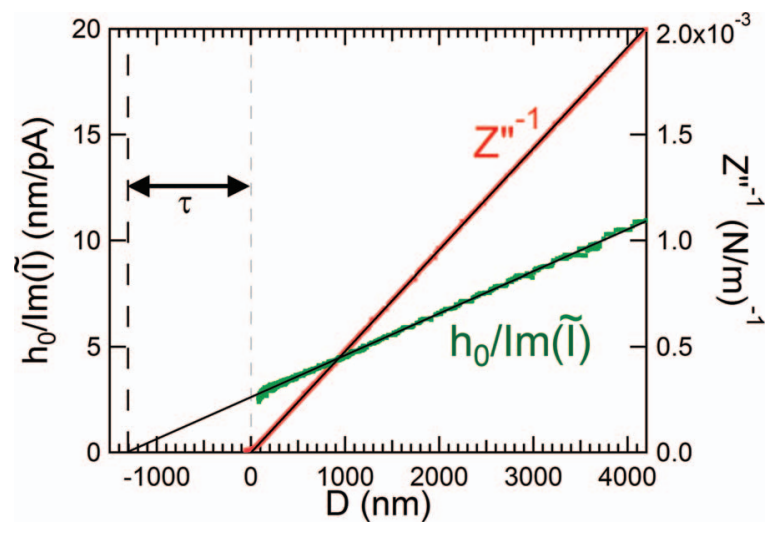

FIG. 4. Comparison of electric and hydrodynamic origins of distances when the plane is coated by a layer of reticulated PDMS (see Eqs. (3) and (7)). Right axis: Inverse of the hydrodynamic impedance $Z^{\prime \prime-1}$ versus surface displacement $D$. The zero on the $x$-axis is determined by fitting $Z^{\prime \prime-1}$ far from the contact by a line. Left axis: Plot of $h_{0} / \operatorname{Im}(\tilde{I})$ for a $5 \mathrm{~V}$ polarization applied between the sphere and plane. The measured thickness of the PDMS layer is $\tau=1324 \pm 2 \mathrm{~nm}$, the real thickness (obtained by taking into account the small difference between the liquid and the thin film dielectric constants) $e=1309 \pm 7 \mathrm{~nm}$. Notice that the main parameter limiting the thickness resolution is the precision in the dielectric constants.

on the dielectric constants, which could be easily improved using a dielectric test fixture.

\section{B. Compatibility with forces measurements}

Once the precision of the conductive interface position measurement is established, an essential point is to verify that this measurement can be conducted without adding significant unwanted forces. Since our SFA is oriented towards dynamic forces measurements, we analyse here the impact of the in situ capacitance measurements on the mechanical impedance $Z(\omega, D)$.

First, we make sure that the imaginary part $Z^{\prime \prime}$ of $Z(\omega$, $D$ ) does not depend on the applied polarization, as expected from Eq. (6). This requires that the viscosity of the Newtonian liquid does not change with the applied electric field. This feature is illustrated in Fig. 3, where values of $Z^{\prime \prime}$ obtained at various polarizations are superimposed. No difference in $Z^{\prime \prime}$ was detected in the range of polarizations studied. It is important to stress that the deviation to the Reynolds damping (Eq. (7)) observed at short distances does not depend on the polarization. It has a purely mechanical origin and is due to elasto-hydrodynamic effects as explained in Ref. 18.

Then, we display in Fig. 5 the real part $Z^{\prime}$ of $Z(\omega, D)$. For a Newtonian liquid between undeformable surfaces, the mechanical part of $Z^{\prime}$ should be zero. But at a polarization of $5 \mathrm{~V}$ we observe negative values of $Z^{\prime}$, which are due to the longrange electrical attractive force. For a polarization of $0.5 \mathrm{~V}$ this attractive stiffness is weaker. Figure 6(a) compares $Z^{\prime}$ to the expected electrical stiffness given by Eq. (6). We indeed observe the linear variation of $Z^{\prime}$ as a function of $1 / D^{2}$, with a slope decreasing with increasing polarizations. This slope is reported in Fig. 6(c) as a function of the square of the polarization. For comparison, we perform the same analysis in Fig. 6(b) with the quasi-static force $F_{\mathrm{dc}}$, expected to scale as $1 / D$ with a slope equal to $-\pi \epsilon R V^{2}$ (see Eq. (4)). The two sets

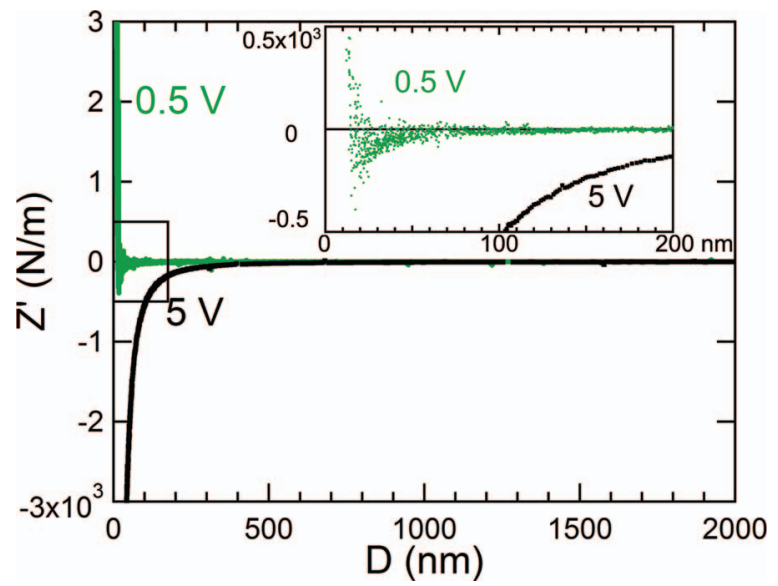

FIG. 5. Real part of the mechanical impedance $Z(\omega, D)=\tilde{F}_{\text {ac }} / h_{0}$ for $0.5 \mathrm{~V}$ and $5 \mathrm{~V}$ polarizations (see Eq. (6)).

of slopes plotted in Fig. 6(c), decrease linearly with the square of the polarization, with a prefactor respectively equal to (215 \pm 5 ) $\mathrm{fF}$ (femtofarad) for the mechanical impedance, and to $(221 \pm 10) \mathrm{fF}$ for the quasi-static force. Both values are very close to the theoretical prediction $\pi \epsilon R=(213 \pm 2) \mathrm{fF}$.

This analysis demonstrates that

(i) the effect of the $0.5 \mathrm{~V}$ polarization on the dynamic impedance is negligible, and

(ii) the effect of all polarizations is fully predicted by the theoretical expression (6), and can therefore be corrected from the measured impedance by a simple subtraction of the electrical stiffness $Z_{\mathrm{EL}}$.

We illustrate hereafter these properties for the measurement of the Young's modulus of a thin elastomer film deposited on the conductive plane (experiment presented in Fig. 4). Here we applied a relatively high polarization (5 V) and measured simultaneously the thickness of the film (as presented in Fig. 4) and the mechanical impedance. The latter is corrected from the electric stiffness $Z_{\mathrm{EL}}$ by subtracting Eq. (6). The residual mechanical impedance is plotted in Fig. 7 and compared to the mechanical impedance measured without polarization. We can see on this figure that there is no measurable difference between these two mechanical impedances, meaning that it is possible to completely erase the effect of the electric field even if it is of several millions of $\mathrm{V} / \mathrm{m}$.

This experiment shows another important application of our distance sensor, which is the non-intrusive measurement of the Young's modulus of an elastic soft layer by the noncontact mechanical impedance. The mechanical impedance of an elastic soft layer supported by a rigid surface is indeed described by the expression ${ }^{19}$

$Z(\omega, D)=\frac{6 \pi \eta R^{2} \omega}{D_{1 / 2}} g_{1 / 2}\left(\frac{D}{D_{1 / 2}}\right), \quad D_{1 / 2}=e\left(\frac{4 \eta \omega}{E}\right)^{1 / 3}$,

where $g_{1 / 2}$ is a master function (tabulated in Ref. 19) and $D_{1 / 2}$ is an elasto-hydrodynamic distance depending on the thickness $e$ of the elastic layer and on its Young's modulus $E$. (Note that Eq. (8) assumes a Poisson ratio $v=0.5$ which is the case 

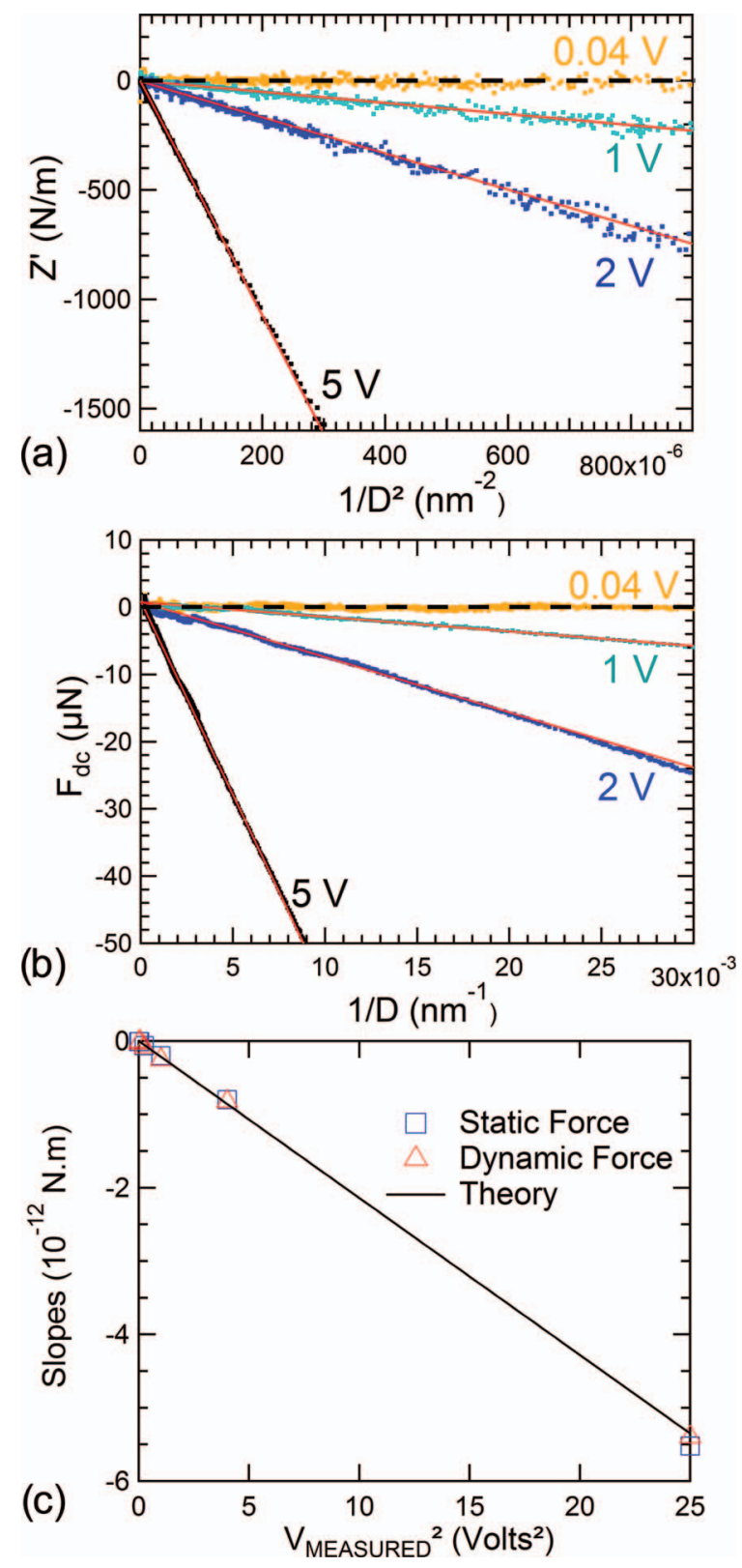

FIG. 6. (a) Real part of the mechanical impedance $Z^{\prime}$ vs. $1 / D^{2}$ for the various polarizations (see Eq. (6)). (b) Quasi-static force as a function of $1 / D$ for the various polarizations (see Eq. (4)). (c) Empty triangles: slopes of the linear variation of $Z^{\prime}$ vs. $1 / D^{2}$ as measured in (a). Empty squares: slopes of the linear variation of the quasistatic force $F_{\mathrm{dc}}$ vs. $1 / D$ as measured in (b). The x-axis is the square of the measured polarizations.

for most elastomers.) Our data were fitted to Eq. (8) using $D_{1 / 2}$ as the only adjustable parameter. An excellent agreement is obtained as can be seen in Fig. 7. More specifically, the feature of $Z(\omega, D)$ which is the most influenced by the thin layer elasticity is the decay of $Z^{\prime}$ as $1 / D^{4}$ at large distances. It is thus essential to obtain a resolution in the measurement of $Z^{\prime}$ better than $10^{-2} \times Z^{\prime \prime}$ for a precise determination of $D_{1 / 2}$. Our SFA allows to determine $D_{1 / 2}=47 \pm 1 \mathrm{~nm}$ in this experiment. The next step is then to extract $E$ from $D_{1 / 2}$. As $E$ depends on $\left(e / D_{1 / 2}\right)^{3}$, an appropriate precision on the layer thickness $e$ at the level of the sphere-plane gap and in the condition of the experiment, is required. From the value $e=1309 \pm 7 \mathrm{~nm}$ measured by our in situ distance sensor, we can safely deter-

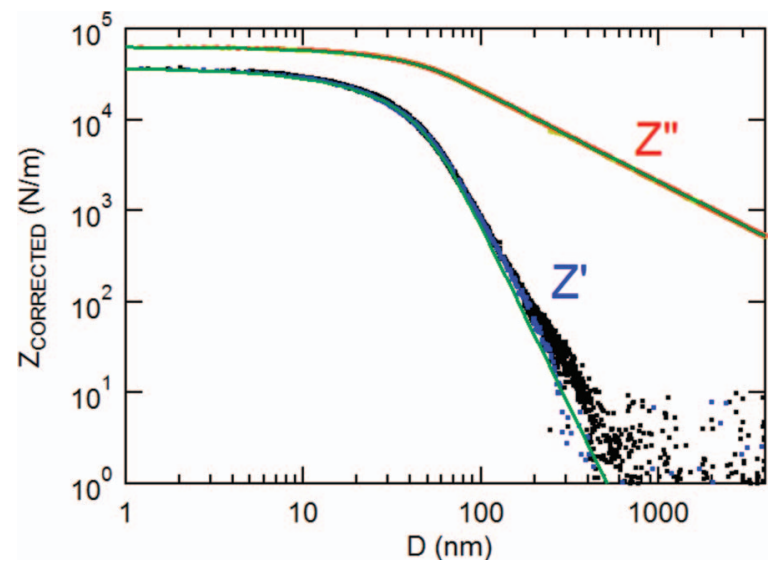

FIG. 7. Real and imaginary parts of the residual mechanical impedance obtained on the thin elastomer film described in Fig. 4. The measured impedance is analysed as explained in the text (see in particular Eq. (8)). Data obtained at a $0 \mathrm{~V}$ polarization (yellow for $Z^{\prime \prime}$ and black for $Z^{\prime}$ curves) and at a $5 \mathrm{~V}$ polarization (red for $Z^{\prime \prime}$ and blue for $Z^{\prime}$ curves) are superimposed. The $Z^{\prime \prime}$ curves are not discernible for all values of $D$, and the $Z^{\prime}$ curves for $D$ smaller than several hundreds of nanometers. These curves are fitted by translating the master function of Eq. (8), here the set of continuous green curves. The best fit displayed here corresponds to $D_{1 / 2}=47 \pm 1 \mathrm{~nm}$. With a measured film thickness $e=1309 \pm 7 \mathrm{~nm}$, a viscosity $\eta=96 \pm 2 \mathrm{mPa} \mathrm{s}$ and a frequency $\omega / 2 \pi=19 \mathrm{~Hz}$, we obtain the Young's modulus of the elastomer film $E=0.99 \pm 0.10 \mathrm{MPa}$.

mine $E=0.99 \pm 0.10 \mathrm{MPa}$ for this micrometric supported elastomer film. It is worth mentioning that such a precision cannot be reached by usual JKR measurements, as shown by Barthel et al. ${ }^{10} \mathrm{We}$ emphasize that the small discrepancy between the measured $Z^{\prime}$ and the theory observed at large distance in Fig. 7, is not due to the electric force, but due to the effect of the elastic compliance of the underlying silicon substrate, that we have not taken into account in Eq. (8) for the sake of clarity (see Refs. 19 and 18).

\section{CONCLUSION}

We presented in this article a simple and reliable method to detect metallic interfaces at distances of several micrometers, with a resolution of about $2 \mathrm{~nm}$ (if the dielectrics constants of the materials studied are known with a sufficient precision), which is of interest for weak force measurements devices. This original setup is very convenient to study buried interfaces and supported thin films, as it provides an in situ measurement of their thickness. The resulting electric forces that could perturb nanorheology or nanomechanics experiments are either negligible, or can be subtracted from the data without altering the measurement precision. Indeed, it has been possible in the presented experiments to remove the electric components in the measured dynamic forces, with an applied electric field of several $\mathrm{MV} / \mathrm{m}$, and to perform noncontact measurements of a thin film Young's modulus.

This simple sensor could be easily adapted on other nanomechanics setups such as the colloidal probe AFM, provided an harmonic oscillation is added to the interacting surfaces. The relevant parameters determining the current amplitude (Eq. (3)) are the product $R f$ of the probe radius and the oscillation frequency, equal to $60 \mathrm{~mm} / \mathrm{s}$ in our setup, and the operating distance range, of several micrometers in the 
SFA. As the distance range is rather hundreds of nanometer in colloidal probe AFM, one expects similar currents for $R f$ $=6 \mathrm{~mm} / \mathrm{s}$, corresponding to an oscillation frequency of order $600 \mathrm{~Hz}$ for a probe radius of $10 \mu \mathrm{m}$. Large AFM tips of the $\mu \mathrm{m}$ could also be used with $\mathrm{kHz}$ frequencies and working distances of tens of nanometers. As the electrical quasi-static and dynamic forces do not depend on frequency, their impact on interaction and mechanical forces should be very similar to the case of the dynamic SFA studied here. With a lateral resolution of order $\sqrt{2 \pi R D}$, this method could also be used to address the spatial distribution of the thickness of dielectric samples.

\section{ACKNOWLEDGMENTS}

This work was conducted with the financial support of ANR Merig.

${ }^{1}$ J. N. Israelachvili and G. E. Adams, J. Chem. Soc., Faraday Trans. 1 74, 975 (1978).

${ }^{2}$ H. Brenner, Chem. Eng. Sci. 16, 242 (1961).

${ }^{3}$ C. Cottin-Bizonne, B. Cross, A. Steinberger, and E. Charlaix, Phys. Rev. Lett. 94, 056102 (2005).
${ }^{4}$ B. Cross, A. Steinberger, C. Cottin-Bizonne, J. Rieu, and E. Charlaix, Europhys. Lett. 73, 390 (2006).

${ }^{5}$ D. Chan and R. Horn, J. Chem. Phys. 83, 5311 (1985).

${ }^{6}$ J.-M. Georges, S. Milliot, J. Loubet, and A. Tonck, J. Chem. Phys. 98, 7345 (1993).

${ }^{7}$ L. Boquet and E. Charlaix, Chem. Soc. Rev. 39, 1073 (2010).

${ }^{8}$ M. Audry, A. Piednoir, P. Joseph, and E. Charlaix, Faraday Discuss. 146, 113 (2010).

${ }^{9}$ P. M. McGuiggan, J. S. Wallace, D. T. Smith, I. Sridhar, Z. W. Zheng, and K. L. Johnson, J. Phys. D: Appl. Phys. 40, 5984 (2007).

${ }^{10}$ E. Barthel, J. Adhes. 83, 729 (2007).

${ }^{11}$ F. Restagno, J. Crassous, É. Charlaix, C. Cottin-Bizonne, and M. Monchanin, Rev. Sci. Instrum. 73, 2292 (2002).

${ }^{12}$ S. Leroy, F. Restagno, and E. Charlaix, Rev. Sci. Instrum. 80, 085103 (2009).

${ }^{13}$ A. Steinberger, C. Cottin-Bizonne, P. Kleimann, and E. Charlaix, Phys. Rev. Lett. 100, 134501 (2008).

${ }^{14}$ S. Leroy, A. Steinberger, C. Cottin-Bizonne, F. Restagno, L. Léger, and E. Charlaix, Phys. Rev. Lett. 108, 264501 (2012).

${ }^{15}$ G. Jourdan, A. Lambrecht, F. Comin, and J. Chevrier, Europhys. Lett. 85, 31001 (2009).

${ }^{16}$ S. de Man, K. Heeck, R. Wijngaarden, and D. Iannuzzi, J. Vac. Sci. Technol. B 28, C4A25 (2010).

${ }^{17}$ L. Boyer, F. Houzé, A. Tonck, J.-L. Loubet, and J.-M. Georges, J. Phys. D: Appl. Phys. 27, 1504 (1994).

${ }^{18}$ R. Villey, E. Martinot, C. Cottin-Bizonne, M. Phaner-Goutorbe, L. Leger, F. Restagno, and E. Charlaix, "Anomalous dynamics in thin Newtonian liquid films: The effect of elastic confinement," Phys. Rev. Lett. (submitted).

${ }^{19}$ S. Leroy and E. Charlaix, J. Fluid Mech. 674, 389 (2011). 